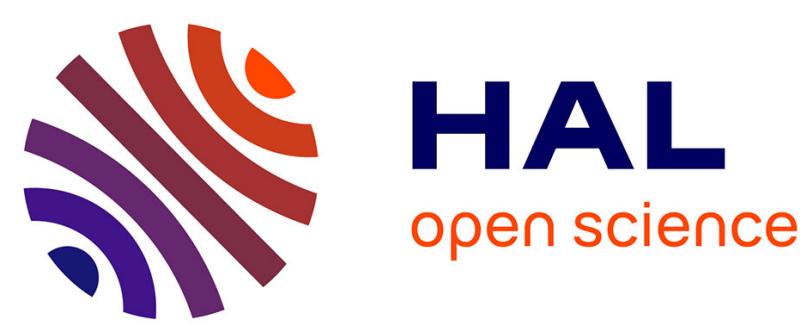

\title{
Adsorption of star polymers
}

\author{
A. Halperin, J. Joanny
}

\section{To cite this version:}

A. Halperin, J. Joanny. Adsorption of star polymers. Journal de Physique II, 1991, 1 (6), pp.623-636. 10.1051/jp2:1991194. jpa-00247545

\section{HAL Id: jpa-00247545 https://hal.science/jpa-00247545}

Submitted on 1 Jan 1991

HAL is a multi-disciplinary open access archive for the deposit and dissemination of scientific research documents, whether they are published or not. The documents may come from teaching and research institutions in France or abroad, or from public or private research centers.
L'archive ouverte pluridisciplinaire HAL, est destinée au dépôt et à la diffusion de documents scientifiques de niveau recherche, publiés ou non, émanant des établissements d'enseignement et de recherche français ou étrangers, des laboratoires publics ou privés. 
Classification

Physics Abstracts

$6125 \mathrm{H}-68.45$

\title{
Adsorption of star polymers
}

\author{
A Halpenn ( ${ }^{1}$ ) and J. F. Joanny $\left({ }^{2}\right)$ \\ (1) Max-Planck-Institut fur Polymerforschung Postfach 3148, W-6500 Manz, F R.G. \\ (2) Institut Charles Sadron, 6, rue Boussingault, F-67083 Strasbourg Cedex, France
}

(Recelved 25 January 1991, accepted 6 March 199I)

\begin{abstract}
Résumé. - Nous étudıons l'adsorptıon de polymères en étoıle sur une surface solıde en utilısant une approche de lois d'échelles basée sur le modèle de blobs de Daoud et Cotton. Pour une étorle formée de $f$ bras contenant chacun $N$ monomères, nous distınguons trois régumes survant la valeur de l'énergie d'adsorption d'un monomère sur la surface $\delta k T$ 1) L'adsorption forte caractérisée par une adsorption complete de tous les bras se produit lorsque $\delta>(f / N)^{3 / 5}$ 2) Une structure en " sombrero" avec $f_{\text {ads }}$ bras adsorbés et $f-f_{\text {ads }}$ bras libres est obtenue si $f^{9 / 20} / N^{3 / 5} \delta<(f / N)^{3 / 5}$

3) Les étolles farblement adsorbées gardent une structure très similaire à celle des étoiles libres en solution Ce régıme exıste si $\delta<f^{9 / 20} / N^{3 / 5}$ La structure correspondant aux étolles faıblement adsorbées peut aussi exıster comme un etat métastable si $\delta>f^{9 / 5} / N^{3 / 5}$
\end{abstract}

\begin{abstract}
The adsorption of star polymers on a flat solid surface is analyzed by means of scaling arguments based on the Daoud-Cotton blob model For the adsorption of a single star, consisting of $f$ arms comprising each $N$ monomers, we distinguish three regimes determined by the adsorption energy of a monomer at the surface, $\delta k T 1$ ) Strong adsorption charactenzed by the full adsorption of all arms occurs for $\delta>(f / N)^{3 / 5}$ 2) A "Sombrero" like structure comprising $f_{\text {ads }}$ fully adsorbed arms and $f-f_{\text {ads }}$ free arms is obtained for $(f / N)^{3 / 5}>\delta>f^{9 / 20} / N^{3 / 5}$ 3) Weakly adsorbed stars retain, essentrally, the structure of a free star This regime occurs for $\delta<f^{9 / 20} / N^{3 / 5}$ The weakly adsorbed structure may also exist as a metastable state if $\delta>f^{9 / 5} / N^{3 / 5}$
\end{abstract}

\section{Introduction.}

The study of polymer adsorption is mostly concerned with linear chains. Much less attention has been given to the adsorption of branched polymers in general and of star polymers in particular Yet, this problem is of interest for practical as well as fundamental reasons The adsorption behavior of star polymers and linear chains differ in an important way The deformation of linear chains upon adsorption is due, solely to the interaction with the surface In marked contrast, the coils comprising the arms of a free star are already deformed because of the repulsive arm-arm interactions $[1,2,3,15]$ Thus, the adsorption of star polymers involves an interplay of two types of deformations, due respectively to molecular architecture and to surface interactions. This balance is of interest because of its impact on the interfacial behavior of stars. The elucidation of star adsorption is also of crucial importance to the understanding of the adsorption of star like micelles $[4,5]$. These are micelles endowed with 
extended coronas which are structurally similar to star polymers. The adsorption of such micelles, when the corona is attracted by the surface, is a possible mechanism for the formation of monolayers of adsorbed block copolymers In turn, these are of practical importance to a variety of applications - colloidal stabilization, compatibilization etc. [6, 7].

In the following we consider the adsorption of star polymers onto a flat surface immersed in a good solvent of low molecular weight The stars are assumed to be monodispersed, comprising $f$ arms such that each arm consists of $N$ monomers Their interaction with the surface is considered as a function of the adsorption energy of a monomer at the surface, $-k T \delta$ We also assume that the core of the star has a small volume and plays no specific role in the adsorption. In particular, the case of a star anchored to the surface because of strong attraction between the core and the surface is not studied.

The next section reviews the relevant results concerning the behavior of free and confined stars. It summarizes the Daoud-Cotton model of free stars and its adaptation to stars confined to slits. This is followed by discussion of single star adsorption. Three regimes occur as $\delta$ is varied. Strong adsorption in which all arms are fully adsorbed, an intermediate regime where only some of the arms adsorb and, finally, weak adsorption where only some of the arms are partly adsorbed. We then present some conjectures on the adsorption isotherm and a few concluding remarks. The two dimensional case, of relevance to simulations, is summarized in the appendix.

\section{Free and confined stars.}

Our description of star polymers is based on the Daoud-Cotton model [1]. The free star is depicted as a spherical region containing close packed blobs arranged in concentric, sphencal shells The blobs in a given shell are of constant size, $\xi(r)$, set by the radius of the shell. Each of the star's $f$ arms is assumed to contribute a single blob to any given shell This is a crucial requirement, allowing for the stretched configurations adopted by densely grafted chains. The volume of a given shell, $r^{2} \xi$, is thus equal to $f \xi^{3}$, the volume of the constituent blobs. Accordingly the correlation length of a free star, $\xi_{\mathrm{f}}(r)$, is given by

$$
\xi_{\mathrm{f}}(r) \sim r / f^{1 / 2} \text {. }
$$

In the Flory approximation, the fractal dimension of a linear chain in a good solvent is $d_{\mathrm{f}}=5 / 3$ and the number of monomers in a blob of size $\xi$ is $(\xi / a)^{5 / 3}$ The local monomer volume fraction is then

$$
\phi(r) \approx f^{2 / 3}(a / r)^{4 / 3}
$$

Monomer conservation determines the radius of the free star, $R_{0}$

$$
R_{0} \approx f^{1 / 5} N^{3 / 5} a .
$$

The $k T$ per blob prescription yields the star free energy $F$ (actually, its excess free energy with respect to $f$ free linear chains consisting each of $N$ monomers)

$$
F / k \dot{T} \approx \int_{R_{\text {core }}}^{R} \xi_{\mathrm{f}}^{-3} r^{2} \mathrm{~d} r \approx f^{3 / 2} \ln \left(R+R_{\text {core }}\right) / R_{\text {core }} \approx f^{3 / 2}
$$

where $R_{\text {core }}$ 1s the radius of the inner, ordered, region comprising the central multi-functional monomer 
A similar approach may be used to stars confined to a slit of width $D$ between two nonadsorbing walls [8] Again, the star is pictured as a layered conglomerate of blobs such that each arm contributes a single blob to any given layer However, the confinement modifies the geometry of the layers and the structure of the blobs. As a result we may distinguish between three regions.

(i) An inner region retaining the structure of a free star, occurs for $r<D$ In this region $\xi$ is given by $\xi_{\mathrm{f}} \sim r / f^{1 / 2}$.

(11) For $r>D$ there occurs an intermediate region charactenzed by loss of the overall spherical symmetry while retaining blobs' three dimensional structure. In particular, the blobs may be considered as spheres of volume $\xi^{3}$, but the shells are now cylindrical. As a result we have $r D \xi \approx f \xi^{3}$ or

$$
\xi_{1} \sim(r D / f)^{1 / 2}
$$

This region extends up to $r \approx f D$ where $\xi_{1} \approx D$ and the confinement begins to affect the blobs structure.

(iii) An extenor, two dimensional region extends beyond $r \approx f D$. It is characterized by cylindrical rather than spherical symmetry. In this region the blobs are two dimensional, of volume $\xi_{\|}^{2} D$, where $\xi_{\|}$is the in-plane correlation length. The Daoud-Cotton construction y1elds $r \xi\|D \approx f \xi\|^{2} D$ or

$$
\xi_{1} \approx r / f
$$

Each two dimensional $\xi_{\|}$blob comprises a string of $g_{\|}$three dimensional blobs of size $D$ set by the slit width. This string exhibits the behavior of a two dimensional self-avoiding random walk 1.e., $\xi_{\sharp} \approx g_{1}^{3 / 4} D$. Accordingly, the total number of monomers in the $\xi_{\|}$blob is $\left(\xi_{\|} / D\right)^{4 / 3}(D / a)^{5 / 3}$ In the limit of large $N$, when the two dimensional region dominates the star behavior, the star in-plane radius, $R_{\|}$, is given by

$$
R_{\|} \sim f^{1 / 4} N^{3 / 4}(a / D)^{1 / 4}
$$

\section{The strong adsorption limit.}

A strongly adsorbed star forms a flat pancake of thickness $D$ (Fig. 1). All $f$ arms are fully adsorbed and the star center is confined within a distance $D$ from the surface. This state is reminiscent of confinement to a slit of width $D$ However, in this case $D$ is not imposed by an external geometric constraint Rather, it is set by the adsorption energy per adsorbed monomer, $-k T \delta$ The free energy of an adsorbed star in this regime consists of two terms.

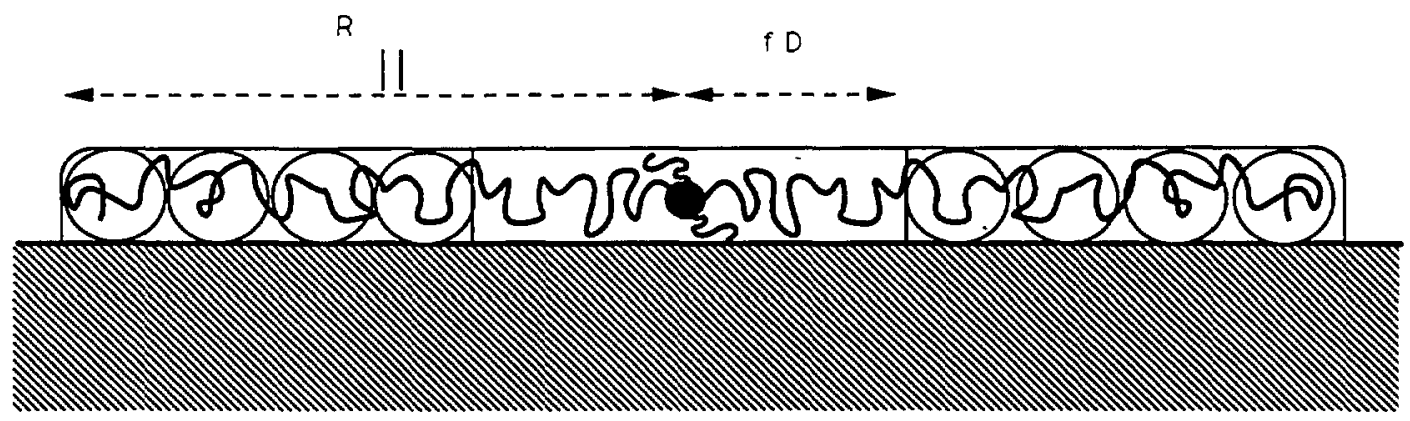

Fig. 1 - Structure of an adsorbed star polymer in the strong adsorption regime. 
The first, $F_{c}$, allows for the deformation of the star and is identical to the confinement free energy of a star in a slit The second term, $F_{\mathrm{a}}$, accounts for the adsorption free energy Clearly, $F_{\mathrm{a}}$ is proportional to the number of monomers in contact with the surface The calculation of this number does not follow the intuitive prescription used in early theories concerned with the adsorption of linear chains These assumed that the monomers are uniformly distributed throughout the layer. Consequently, the total number of monomers per unit area within a layer of thickness $z(a \leqslant z \leqslant D)$ scales as $z / D$ and the fraction of monomers at the surfaces scales as $a / D$ In time it was recognized $[9,10]$ that this approach must be modified to allow for the fact that each monomer at the surface drags its neighbors with it. As a result the monomer volume fraction within a layer of thickness $z$ is not constant but decays algebraically as $(z / D)^{m}$ with an exponent $m \approx 1 / 3$. This picture may be reformulated in terms of adsorption blobs [11]. These are isotropic chain segments of size $D$ exhibiting the behavior of a free coll Thus, in a good solvent each blob consists of $g \approx(D / a)^{5 / 3}$ of monomers. In each adsorption blob, only $g^{\phi}$ of the $g$ monomers are in contact with the surface where $\phi=1+(m-1) / d_{f} \approx 3 / 5$ is the crossover exponent. The adsorbed state of a single linear chain may be fully described in terms of such blobs It is pictured as a self avoiding, two dimensional string of $D$ blobs As we shall see the situation in our case is more complicated because of the star topology and the resulting interactions between the arms. For brevity we first analyze the confinement free energy.

The confinement free energy is propertional to the overall number of blobs in the three "stellar » regions described in the previous section. The number of blobs in a given region is found by integrating the appropriate blob density In the exterior region one must account for two types of blobs $D$ blobs arising from the confinement of each arm and two dimensional $\xi_{\|}$blobs reflecting the interaction between the $f$ arms which form a two dimensional star Altogether we find

$$
\begin{aligned}
& F_{\mathrm{c} / k T} \approx \int_{r_{\text {corc }}}^{D} \xi_{\mathrm{f}}^{-3} r^{2} \mathrm{~d} r+\int_{D}^{f D} \xi_{i}^{-3} r D \mathrm{~d} r+\int_{f D}^{R}\left[1+\left(\xi_{\|} / D\right)^{4 / 3}\right]\left(\xi_{\|}^{2} D\right)^{-1} r D \mathrm{~d} r \\
& F_{\mathrm{c} / k T} \approx f^{3 / 2} \ln \left(D / r_{\text {core }}\right)-f^{3 / 2}+f^{2} \ln \left[f^{-3 / 4}(a / D)^{5 / 4} N^{3 / 4}\right]+f(a / D)^{5 / 3} N
\end{aligned}
$$

Since the last two terms are clearly dominant we will use

$$
F_{\mathrm{c} / k T} \approx f N(a / D)^{5 / 3}+f^{2}
$$

where the logarithmic factor multiplying $f^{2}$ has been approximated by a constant.

Only three dimensional blobs in contact with the surface are relevant to the adsorption free energy, $F_{\text {a }}$ Each blob, comprising $g$ monomers, contains $g^{\phi}$ monomers in contact with the surface The corresponding contribution to $F_{\mathrm{a}}$ is thus $-g^{\phi} k T \delta$. Accordingly, the adsorption free energy due to the two interior regions is obtained by summing the contributions due to all surface grazing adsorption blobs

$$
-F_{\mathrm{a} / \delta k T} \approx \int_{D}^{f D}\left(\xi_{\mathrm{l}} / a\right) \xi_{1}^{-2} r \mathrm{~d} r+\int_{f D}^{R}(D / a)\left(\xi_{\|} / D\right)^{4 / 3} \xi_{\|}^{-2} r \mathrm{~d} r \approx \delta f N(a / D)^{2 / 3}
$$

Altogether, the stars free energy, $F=F_{\mathrm{a}}+F_{\mathrm{c}}$ is given by

$$
F / k T \approx f^{2}+f N(a / D)^{5 / 3}-\delta f N(a / D)^{2 / 3} .
$$

Minimization with respect to $D$ yields

$$
D \approx \delta^{-1} a,
$$


a result familiar from the study of linear adsorbed chains [12]. This reflects the dominance of the external region contribution to $F$ which is due primarly to single arm behavior By combining the last two results we obtain the total free energy of a strongly adsorbed star

$$
F / k T \approx f^{2}-f N \delta^{5 / 3} \text {. }
$$

The strong adsorption limit is dominated by the contributions of the external region Clearly this description is only meaningful if the external region of the star indeed exusts i.e. $R>f D$ This condition defines cross over values of $D$ and $\delta, D_{\mathrm{c}}=R_{\Downarrow} / f$ and $\delta_{\mathrm{c}}=a / D_{\mathrm{c}}$ given by

$$
D_{\mathrm{c}} \approx(N / f)^{3 / 5} a, \quad \delta_{\mathrm{c}} \approx(f / N)^{3 / 5}
$$

For $\delta<\delta_{\mathrm{c}}$ or $D>D_{\mathrm{c}}$ the strong adsorption limit can no longer be obtained and the absorbed star adopts the «Sombrero » configuration discussed in the next section.

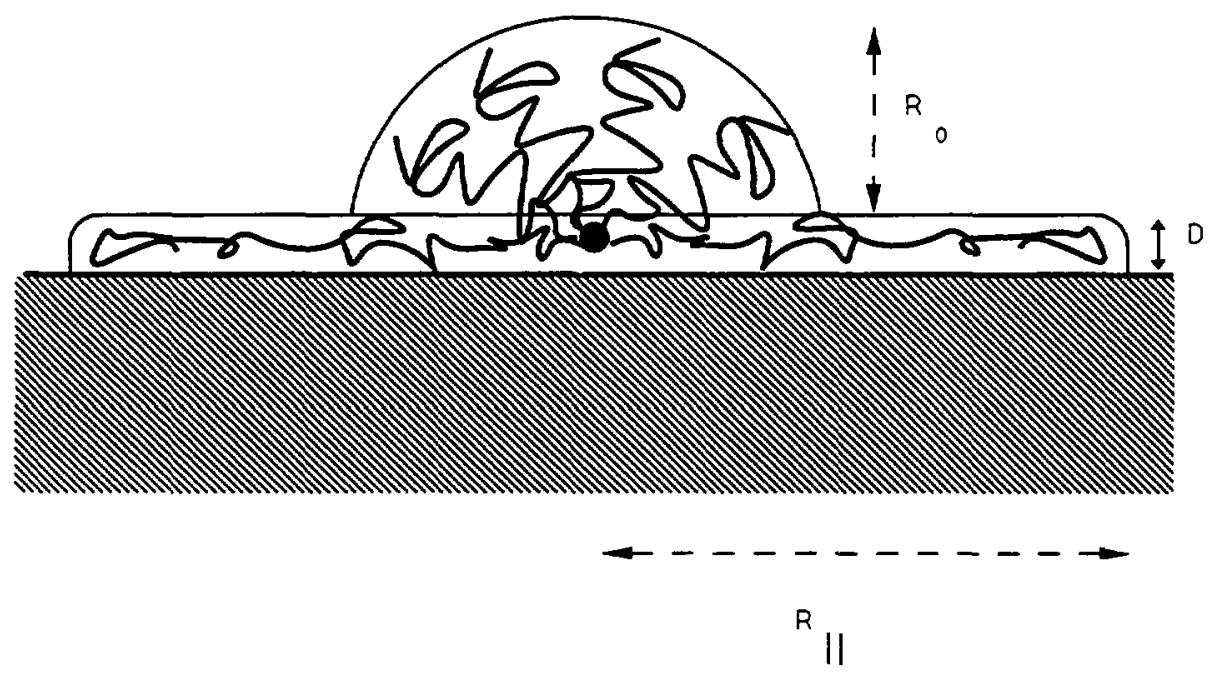

Fig 2 - Structure of an adsorbed star in the «Sombrero » regime

\section{The Sombrero structure.}

When $\delta$ falls below $\delta_{c}$ the flat pancake structure, characteristic of the strong adsorption regime, is replaced by a "Sombrero" configuration (F1g. 2). In this regime only $f_{\text {ads }}<f$ arms are adsorbed. These form a confined star structure of the type considered in the previous section $1 \mathrm{e}$, a flat pancake, of thickness $D \sim \delta^{-1} a$, incorporating the star center. The remaining $f_{\text {free }}=f-f_{\text {ads }}$ arms are free These adopt a free star configuration but occupy a hemisphere rather than a sphere The free arms are stretched according to the DaoudCotton model As we shall see the radius of the adsorbed layer is much larger than that of the hemisphere hence the name «Sombrero». The free energy of this structure contains two terms, $F_{\text {free }}$ and $F_{\text {ads }}$, accounting respectively for the contributions of the free and adsorbed arms. $F_{\text {free }}$ is given by (34) with $f_{\text {free }}$ replacing $F F_{\text {ads }}$ is obtained from (3.8) once $f_{\text {ads }}$ takes the place of $f$. Accordingly, $F$ is given by

$$
F / k T \approx f_{\text {free }}^{3 / 2}+f_{\text {ads }}^{2}-f_{\text {ads }} N \delta^{5 / 3} .
$$


The minimization with respect to $f_{\text {ads, }}$ which amounts to equating the chemical potential of the free and the adsorbed arms, leads to

$$
\left(f-f_{\text {ads }}\right)^{1 / 2}-f_{\text {ads }}+N \delta^{5 / 3}=0
$$

For $f^{1 / 2} \ll f_{\text {ads }} \leqslant f$ the first term is negligible and $f_{\text {ads }}$ is given by

$$
f_{\text {ads }} \approx N \delta^{5 / 3} \text {. }
$$

This result is also obtained if we use the criterion $D=D_{\mathrm{c}}$ to set the number at adsorbed arms. In other words the adsorbed layer thickness is maintained at $D$ by desorption of arms and $D \approx\left(N / f_{\mathrm{ads}}\right) a^{3 / 5}$ leads to $f_{\mathrm{ads}} \approx N /(D / a)^{5 / 3} \approx N \delta^{5 / 3}$

The horizontal radius of the star is obtained from equation (27)

$$
R_{\|} \approx N \delta^{2 / 3}
$$

or $R_{\|} \approx f_{\text {ads }} D . R_{\|}$is indeed larger than the radius of the hemispheres, $R \approx f_{\text {free }}^{1 / 5} N^{3 / 5} a$, provided $D=\delta^{-1} a<\xi_{\mathrm{M}}$ where $\xi_{\mathrm{M}} \sim N^{3 / 5} f_{\text {free }}^{-3 / 10}$ is the size of the largest blob of the DaoudCotton structure in the hemisphere Equation (4.3) also determines the free energy of the «Sombrero » structure

$$
F / k T \approx-N^{2} \delta^{10 / 3}+f^{3 / 2} \approx-R_{\|}^{2} / D^{2}+f^{3 / 2} .
$$

This free energy is positive if $\delta<f^{9 / 20} N^{-3 / 5}$ and becomes equal to the free energy of a free star confined in a half space at small values of $\delta$. The Sombrero structure exists only at intermediate values of the adsorption energy $\delta$. $\delta$ must be smaller than $\delta_{\mathrm{c}}$ but we also required $f_{\text {ads }} \gg f^{1 / 2}$ i e., $\delta>N^{-3 / 5} f^{3 / 10}$ In terms of the pancake thickness $D$, the sombrero exists if $D_{\mathrm{c}}<D<\xi_{\mathrm{M}}$. If $D>\xi_{\mathrm{M}}$ the star is only very weakly adsorbed, a situation we do not consider further

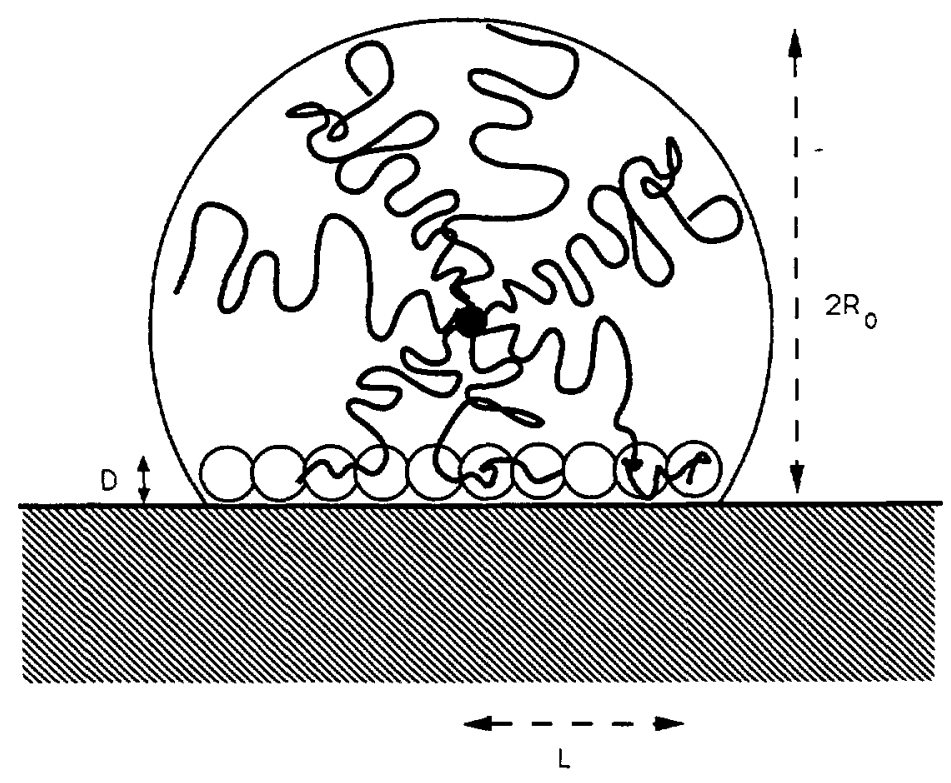

Fig 3 - Structure of an adsorbed star in the weak adsorption limit. 


\section{Weakly adsorbed stars.}

For sufficiently small adsorption energy, $\delta$, the adsorbed star is only weakly deformed only some of the arms are partly adsorbed. In this limit it is helpful to view the star as a "droplet » of a semidilute solution The adsorbed monomers are not expected, thus, to develop a two dimensional Daoud-Cotton structure. Rather, they form a uniform semidilute surface layer The adsorbed monomers originate in a small spherical cap characterized by an apex angle of $2 \theta_{0} \ll \pi / 2$ (Fig. 3). The boundaries of the cap and the surface layer are assumed to coincide $1 \mathrm{e}$, the layer in-plane radius, $L$, is given by $L \approx R \sin \theta_{0} \approx R \theta_{0}$. The number of partly adsorbed arms, $f_{\text {ads }}$, is thus $f_{\text {ads }} \approx f \theta_{0}^{2}$ The remainder of the star is unperturbed. A full characterization of this state is possible if one assumes, as in the case of a semidilute solution, that the adsorbed layer consists of close packed $D$ blobs. The area of the adsorbed layer, $L^{2} \approx R^{2} \theta_{0}^{2}$, is than equal to the total area of the $D$ blobs, $(n / g) D^{2}, n$ being the number of adsorbed monomers This requirement determines $\theta_{0}$ and allows for a complete characterization of this adsorption regime In order to carry out this program it is necessary to find the number of adsorbed monomers $n$, or equivalently, the number of monomers in the sphencal cap For $\theta_{0} \ll \pi / 2$, the regime of interest, $n$ is given by

$$
n \approx f N \theta_{0}^{4} \text {. }
$$

Accordingly

$$
\theta_{0} \approx f^{-3 / 10} N^{1 / 10}(a / D)^{1 / 6} \approx f^{-3 / 10} N^{1 / 10} \delta^{1 / 6}
$$

The number of adsorbed monomers is thus

$$
n \approx f^{-1 / 5} N^{7 / 5} \delta^{2 / 3}
$$

$L$ is given by

$$
L \approx R_{0} \theta_{0} \approx f^{-1 / 10} N^{7 / 10} f^{1 / 6}
$$

and the number of partly adsorbed arms is

$$
f_{\mathrm{ads}} \approx f \theta_{0}^{2} \approx f^{2 / 5} N^{1 / 5} \delta^{1 / 3}
$$

Each of these arms contributes $N_{\text {ads }}$ monomers to the adsorbed layer

$$
N_{\mathrm{ads}} \approx n / f_{\mathrm{ads}} \approx f^{-3 / 5} N^{6 / 5} \delta^{1 / 3}
$$

Within this picture the free energy of a weakly adsorbed star is

$$
F / k T \approx f^{3 / 2}-(n / g) g^{\phi} \delta \approx f^{3 / 2}-f^{-1 / 5} N^{7 / 5} \delta^{7 / 3}
$$

The first term, the free energy of a free star, approximates the free energy of the unperturbed region of the adsorbed star. It is supplemented by the adsorption free energy of the $n$ monomers which may be written as $-k T L^{2} / D^{2}$.

One may gain some insight by considering a different route to these results It calls for minimization of an appropriate free energy To account for the contributions of the adsorbed layer we view it as a close packed array of two dimensional blobs of size $\xi_{\|}$Each $\xi_{\|}$blob comprises a self avoiding, two dimensional string of $D$ blobs. The monomer area fraction within the adsorbed layer is $\Gamma \approx n a^{2} / L^{2} \approx f^{3 / 5} N^{-1 / 5} \theta_{0}^{2}$. This is also the monomer 
area fraction within a $\xi_{\|}$blob. Since the number of $D$ blobs within a $\xi_{\rrbracket}$ blob is $\left(\xi_{\|} / D\right)^{4 / 3}$ we have $\Gamma \approx\left(\xi_{\|} / D\right)^{4 / 3}(D / a)^{5 / 3}\left(a / \xi_{\|}\right)^{2}$. Accordingly, $\xi_{\|} / a \approx f^{-9 / 10} N^{3 / 10} \delta^{-1 / 2} \theta_{0}^{-3}$ while the number of $\xi_{\|}$blobs is $n_{\|} \approx L^{2} / \xi_{\|}^{2} \approx f^{11 / 5} N^{3 / 5} \delta \theta_{0}^{8}$. The total number of $D$ blobs is thus $n_{D} \approx\left(\xi_{\|} / D\right)^{4 / 3} n_{\|}$or $n_{D} \approx f N \delta^{5 / 3} \theta_{0}^{4}$. This completes the description of the adsorbed layer However, for completeness it is necessary to allow for the fact that the $n$ adsorbed monomers are no longer part of the Daoud-Cotton structure. Consequently, the total number of blobs in the unperturbed region of the star is $f^{3 / 2}-n_{\text {blob, }}$, where $n_{\text {blob }}$ is the number of blobs in the spherical cap giving rise to the adsorbed layer. $n_{\text {blob }}$ is given by

$$
n_{\text {blob }} \approx f^{3 / 2} \theta_{0}^{4}
$$

Altogether we have

$$
F / k T \approx\left(f^{3 / 2}-n_{\text {blob }}\right)-n_{D} g^{\phi} \delta+n_{\|}
$$

or

$$
F / k T \approx f^{3 / 2}\left(1-\theta_{0}^{4}\right)-f N \delta^{5 / 3} \theta_{0}^{4}+f^{11 / 5} N^{3 / 5} \delta \theta_{0}^{8} .
$$

The first term is the number of blobs in the unperturbed regions of the star. This is the free energy of the regons retaining the free star structure. The second term accounts for the confinement of the adsorbed chain segments and for the adsorption energy. The third term reflects the excluded volume due to interactions between the adsorbed chain segments. Upon minimization with respect to $\theta_{0}$ we recover (5.3) In turn, this leads to $\xi_{\|}=D$ as was assumed earlier.

The weak adsorption structure proposed here is possible only if $\theta_{0} \ll 1$ or if the adsorption energy $\delta$, is smaller than $f^{9 / 5} N^{-3 / 5}$. Thus, if we ignore the very weak adsorption obtained when $D>\xi_{\mathrm{M}}$, we expect the weak adsorption structure when $N^{-3 / 5} f^{-9 / 5} a<D<\xi_{\mathrm{M}}$. Within this range, both the Sombrero and the strong adsorption structures are possible and one must" compare the free energies to determine the equilibrium configuration If $D<D_{\mathrm{c}}$ (or equivalently $\delta>\delta_{c}$ ), the strong adsorption structure is stable If $D_{c}<D<N^{3 / 5} f^{-9 / 20} a$ the energy of the Sombrero structure is lower than the energy of a weakly adsorbed star and the Sombrero structure is stable If $N^{3 / 5} f^{-9 / 20}<D<\xi_{M}$, the dominant contribution in the free energy of these two structures is that of the free arms. In the Sombrero construction the free arms are confined in a half space, while in the weak adsorption construction they are not constrained and the free energy is lower; the stable structure corresponds thus to weak adsorption.

Although the weak adsorption structure is stable only in a small range of adsorption energies, it may exist in a much broader range as a metastable state. One can expect that in many cases an adsorbing star remains in the weak adsorption configuration and has a large potential barrier (of order $k T f^{3 / 2}$ ) to overcome in order to adsorb as a flat pancake. This suggests strong hysteretic effects in the adsorption of star polymers

\section{A little on concentration effects.}

The adsorption of a single chain is a somewhat academic problem that cannot be studied expenmentally. Rather, the relevant expenmental quantity is the adsorption isotherm. The underlying physics are qualitatively clear. The system strives for maxımum surface coverage with the minimal possible deformation of the adsorbing polymers in order to minimize its interfacial energy. Thus, linear chains at saturation attain complete coverage while forming loops and tails so as to minimize their deformation [12,13] Adsorbed stars in contact with a 
reservoir are capable of ncher behavior In particular, the deformation may be relieved either by arm desorption or by loop formation Intuitively, arm desorption is expected to be dominant in the vicinity of the weak adsorption regime In this regime the deformation penalty associated with the star topology determines the behavior Loop formation is anticipated in the strong adsorption regime where surface induced deformations are dominant In general both processes may come into play simultaneously A complete theory, accounting for the two contributions, is beyond the scope of this work because it would require a description of the star much more precise than the Daoud-Cotton blob model It is, however, possible to focus on situations involving only arm desorption This approach is expected to lead to a realistic adsorption isotherm in the weak adsorption limit. While a similar analysis for the regimes of stronger adsorption is probably misleading, it may provide some insight Accordingly, we construct adsorption 1sotherms for all regimes assuming that no loop formation takes place

To construct the adsorption isotherm we equate the chemical potential of the free stars in the solution, $\mu_{b}$, to the chemical potential of the stars in the adsorbed layer, $\mu$. $\mu_{\mathrm{b}}$ is given by

$$
\mu_{0} / k T \approx f^{3 / 2}+\ln c_{\mathrm{b}} R_{0}^{3} / f N
$$

where $R_{0} \approx f^{1 / 5} N^{3 / 5} a, c_{b}$ is the bulk concentration and $f^{3 / 2} k T$ is the excess configurational free energy of a star with respect to $f$ free linear chains comprising each $N$ monomers. The second term accounts for the translational entropy of a dilute gas of stars viewed as hard spheres of radius $R_{0}$ The adsorbed layer is characterized by a surface excess of $\Gamma$ adsorbed monomers per unit area or by $\sigma=\Gamma / f N$, the number of adsorbed stars per unit area Denoting the free energy per unit area by $\tilde{F}$, the chemical potential of an adsorbed star is $\mu=\partial \tilde{F} / \partial \sigma$.

We first consider the weak adsorption isotherm The chemical potential of a dilute surface of weakly adsorbed stars is

$$
\mu / k T \approx \ln \sigma R_{0}^{2}+f^{3 / 2}-f^{-1 / 5} N^{7 / 5} \delta^{7 / 3}
$$

where the first term accounts for the two dimensional translational entropy and the remaining terms, given by (5 8), specify the configurational free energy of a single weakly adsorbed star. Of these two, the last gives the adsorption free energy $\Delta F_{\text {ads }} / k T \approx-L^{2} / D^{2}$ By balancing the two chemical potentials we obtain

$$
\sigma \approx \frac{c_{\mathrm{b}} R_{0}}{f N} \exp \left(-\frac{\Delta F_{\mathrm{ads}}}{k T}\right)=\frac{c_{\mathrm{b}} R_{0}}{f N} \exp \left(f^{-1 / 5} N^{7 / 5} \delta^{7 / 3}\right)
$$

Since weakly adsorbed stars are barely deformed, close packing is attained for $\sigma \approx 1 / R_{0}^{2}$ corresponding to a continuous surface layer of weakly distorted stars of radius $R_{0}$. This condition is obtained for bulk concentration of

$$
c_{\mathrm{b}}^{\mathrm{cp}} \approx \frac{f N}{R_{0}^{3}} \exp -\left(f^{-1 / 5} N^{7 / 5} \delta^{7 / 3}\right)
$$

As the weak adsorption regime is found for $\delta<f^{9 / 20} N^{3 / 5}, c_{\mathrm{b}}^{\mathrm{cp}}$ is larger than $c^{*} \exp \left(-f^{17 / 20}\right)$ and is well in the dilute regime Above this close packing concentration we expect a plateau in the adsorption isotherm, at least for dilute star solutions

This analysis is easily extended to the dilute surface region of the adsorption isotherms characterizing the stronger adsorption regimes The first term of $\mu / k T$, $\ln \sigma R_{0}^{2}$, must be 
replaced by $\ln \sigma R_{\mathrm{s}}^{2}$ where $R_{\mathrm{s}}$ is the effective horizontal radius of the adsorbed star If the surface concentration, $\sigma$, is small enough $R_{\mathrm{s}}$ is the radius of an isolated adsorbed star given by equation (27) in the strong adsorption regime and by equation (4.4) in the Sombrero regime. For higher values of $\sigma$ the adsorbed stars interpenetrate and $R_{\mathrm{s}}$ must be determined selfconsistently.

For the Sombrero regime the free energy of the adsorbed star is given by (4.5) and consequently

$$
\mu / k T=\ln \sigma R_{s}^{2}+f^{3 / 2}-N^{2} \delta^{10 / 3}
$$

leading to

$$
\sigma \approx \frac{c_{\mathrm{b}} R_{0}^{3}}{f N R_{\mathrm{s}}^{2}} \exp \left(N^{2} \delta^{10 / 3}\right)
$$

The free energy of strongly adsorbing stars is given by (3 6) leading to

$$
\mu / k T=\ln \sigma R_{\mathrm{s}}^{2}+f^{2}-f N \delta^{5 / 3}
$$

and to

$$
\sigma \approx \frac{c_{\mathrm{b}} R_{0}^{3}}{f N R_{\mathrm{s}}^{2}} \exp \left(f^{3 / 2}-f^{2}+f N \delta^{5 / 3}\right)
$$

In all cases the dilute surface 1sotherm is of the form $\sigma \sim c_{\mathrm{b}} \exp \left(-\Delta F_{\text {ads }} / k T\right)$ where $\Delta F_{\text {ads }}$ is the difference between the free energies of an adsorbed and of free stars At this point, it is interesting to compare the adsorption of stars to the adsorption of linear chains and to discuss the competition between the adsorption of two types of polymers.

For a linear chain comprising $M=f N$ monomers

$$
\Delta F_{\mathrm{d}} / k T=-M(a / D)^{5 / 3}
$$

In the case of a linear chain with the same number of monomers as the star, $M=f N$, the adsorption energy $\Delta F_{\mathrm{c}}$ is always larger (in absolute value) than the adsorption energy of a star (Eqs. (3.6), (4.5) or (58)) The adsorption of linear chains is thus favored compared to that of stars of same molecular weight and same chemical nature. This is due to the softer elasticity of the linear chans

Experimentally one thus expects preferential adsorption of linear chains from a solution containing both types of polymers and the displacement of adsorbed stars by linear chains. These predictions are made here for very small values of the bulk concentration, however, we expect them to remain qualitatively true for any bulk concentration. The role of the star core was systematically ignored in our discussion. However, one should note that a very strong adsorption of the core at the surface can considerably increase the adsorption of the stars.

Further extension of this analysis to semidilute and saturated surfaces is difficult since a proper description must account for loop formation as well as arm desorption. Nevertheless one may gain some insight by considering a fictious system which does not form loops In particular we focus on a semidilute surface in the strong adsorption regime However, it must be stressed that the following discussion is almost certainly incomplete since it only allows for arm desorption.

At $\sigma \approx 1 / R_{\mathrm{s}}^{2} \approx f^{-1 / 2} N^{-3 / 2} \delta^{-1 / 2}$ the surface is contınuously covered by non interpenetration stars However, the coverage in the extenor region of each star is incomplete since the two dimensional Daoud-Cotton structure involves $\xi_{\|}$blobs comprising self-avoiding strings of 
adsorption blobs. Further increase in $\sigma$ causes interpenetration and compression of the coronas. The associated screening of excluded volume interactions destroys the DaoudCotton structure leading, eventually, to a contınuous coverage by $D$ blobs. This is attained when the horizontal radius of the star is given by $R_{\|}^{2} \approx\left(f N / g_{D}\right) D^{2} \approx f N(D / a)^{1 / 3} a^{2}$ The corresponding surface coverage is

$$
\sigma_{\mathrm{c}} \approx 1 /(f N) \delta^{1 / 3} a^{2}
$$

Beyond $\delta_{\mathrm{c}}$, further increase in $\sigma$ must be accompanied by arm desorption. For higher coverage $R_{\mathrm{s}}^{2} \approx f_{\text {ads }} N \delta^{1 / 3} a^{2}$ or, since $\sigma \approx 1 / R_{\mathrm{s}}^{2}$,

$$
f_{\mathrm{ads}} \approx 1 /(N \sigma) \delta^{1 / 3} a^{2} \approx f \sigma_{\mathrm{d}} / \sigma
$$

To proceed further we must specify the chemical potential of an adsorbed star in this regime The free energy per star is simply

$$
F / k T=-R_{\mathrm{s}}^{2} / D^{2}+\left(f-f_{\mathrm{a}}\right)^{3 / 2} .
$$

Accordingly, the free energy per unit area, $\tilde{F}$, is

$$
\tilde{F} / k T \approx-D^{-2}+f^{3 / 2} \sigma\left(1-\sigma_{\mathrm{c}} / \sigma\right)^{3 / 2}
$$

and the chemical potential $\mu=\partial \tilde{F} / \partial \sigma$ is

$$
\mu / k T \approx f^{3 / 2}\left(1-\sigma_{c} / \sigma\right)^{1 / 2}\left(1+\sigma_{c} / 2 \sigma\right)
$$

The adsorption isotherm is obtained by equating the surface and the bulk chemical potentials. The concentration where close packing on the surface is reached is of order $c^{\mathrm{cp}} \sim c^{*} \exp -f N(a / D)^{5 / 3}$ and is thus extremely dilute. At higher bulk concentrations, but still in the dilute range, the adsorption ssotherm reaches a plateau of order $\sigma_{c}$ where it varies only loganthmically with the bulk concentration. The surface excess is then $\Gamma=\sigma_{\mathrm{c}} f N \sim D^{-1 / 3}$ and is of the same order as what it would be for adsorbed linear chains.

One should note that arm desorption results in a Sombrero like structure which is consistent only if the radius of the base, $R_{i \mid}$, is larger than the radius of free stars. This arm desorption mechanism is thus possible only in the very strong adsorption regime where $D<N^{3 / 5} f^{-9 / 5} a$. By increasing the concentration in this regime well above $c^{\mathrm{cp}}$ one could eventually form an adsorbed layer comprising close packed hemispheres of size $R_{0}$.

In the Sombrero regime a close packing of $D$ blobs on the surface is reached when $\sigma \sim 1 / R^{2}$ where the radius $R_{\|}$is given by equation (44) leading to $\sigma=N^{-2} \delta^{-4 / 3} \sim 1 /\left(f_{\text {ads }} N\right) \delta^{1 / 3}$ This, as well is obtaned for extremely low bulk concentrations of order $c^{*} \exp \left(-N^{2} / D^{10 / 3}\right)$. Here, again, we have no precise description of the adsorbed layer at higher concentrations

\section{Concluding remarks.}

We have discussed the adsorption of star polymer solutions on flat solid surfaces. Although our study has been concerned primarily with single star adsorption, several conclusions can be drawn that can be of some experimental relevance.

- If the solution is extremely dilute different stars adsorb independently and may adopt one of the three structures studied in sections 4, 5 and 6 1.e., the strong adsorption, Sombrero, and weak adsorption structures

- Although the weak adsorption structure is thermodynamically stable in a small range of 
adsorption energies, it may be found as a metastable state over a much broader range. In such metastable states the stars do not spread on the surface and essentially remain undeformed spherical objects In such cases the thickness of the adsorbed layer is of the order of magnitude of the radius of a free star This could explain recent experimental results on the effect of starlike micelles on collordal stability [7]

- The adsorption of linear polymers is favored with respect to that of starlike polymers. We expect this to be a general result valid not only for starlike polymers but also for any kind of branched polymer and in particular for a randomly branched polymer obtained in a gelation process

Our results on single star adsorption can also be tested by numerical simulations. Such simulations are actually in progress in Mainz [14] and we include an appendix on the extension of our results to two-dimensional stars, on which some of the simulations are performed.

However, the description of star polymers provided by the Daoud-Cotton blob model is rather rough and our work raises several unanswered questions

- We were not able to give a full description of the plateau region of the adsorption isotherm. A quantitative understanding of the adsorption requires the elucidation of the relative roles of arm desorption and loop formation

- We only considered dilute star solutions In a semidilute solution the ends of the star arms are essentially free and behave as linear chains. They then adsorb more easily than the central parts of the stars Well in the semidilute regime, one can thus expect an adsorbed layer with the same characteristics as an adsorbed semidilute solution of linear chains. All the star centers are expelled from this adsorbed layer. However, the crossover to the dilute solution case discussed here is not understood.

- The possibility of metastable adsorbed states and hysteretic effects also raises questions concerning the nature of the special transition between adsorbed and desorbed stars.

Finally, as we noted earlier, it seems that the most important example of star polymer adsorption is provided by starlike micelles endowed with extended, adsorbing coronas The main difference is that these micelles are not permanent objects and may change their nature upon adsorption. We plan to discuss their adsorption behavior in more detall in a future work

\section{Acknowledgements.}

A. $\mathrm{H}$ acknowledges the generous hospitality of Prof. E W Fischer and the Max-PlanckInstitut für Polymerforschung.

\section{Appendix. Adsorption in two dimensions.}

The adsorption behavior of stars is simpler in two dimensions The strong adsorption regime is unattainable because chains may not cross each other once two arms adsorb, the surface is shielded and other arms can not approach it Consequently, the two possible scenarios comprise the weak adsorption and the sombrero structures. Furthermore, the Daoud-Cotton construction is achievable only for the free arms. The adsorbed arms interact with a line where such structures may not occur. Apart from these simplifying features the two dimensional case is distinct because the exponents assume different values In particular [16], $\nu \approx 3 / 4$ and $\phi \approx 1 / 2$. Accordingly, $\delta_{\mathrm{c}} \sim N^{-1 / 2}, D / a \sim \delta^{-3 / 2}$ and $g_{\mathrm{a}} \sim \delta^{-2}$

We first outline the Daoud-Cotton model for two dimensions. The requirement that any arm contributes a single blob to each shell leads to $r \xi \approx f \xi^{2}$ or

$$
\xi \approx r / f
$$


Since the two dimensional $\nu$ is $\nu \approx 3 / 4$, the number of monomers per blob, $g$, obeys $\xi \approx g^{3 / 4} a$ leading to $g \approx(r / a f)^{4 / 3}$ The monomer area fraction, $\phi \approx f a^{2} / \xi^{2}$ is accordingly

$$
\phi \approx(a f / r)^{2 / 3}
$$

Conservation of monomers leads thus to

$$
R_{0} / a \approx f^{1 / 4} N^{3 / 4}
$$

The total free energy of the star is given by

$$
F / k T \approx f \int_{r_{\mathrm{c}}}^{R_{0}} \xi^{-1} \mathrm{~d} r \approx f^{2} \ln \left(R_{0} / r_{\mathrm{c}}\right) \approx f^{2} .
$$

As noted earlier, the confinement problem is irrelevant to the adsorption of stars in two dimensions. Let us just note the central features of a star confined to a slit of width $D$ in two dimensions. In this case we may distinguish between two stellar regions. An inner region which retains the Daoud-Cotton structure of a free star occurs for $r<D$ For $r>D$ the star behaves as a two dimensional flat grafted layer In this region $\xi$ and $\phi$ are position independent. In particular $\xi \approx D / f$ and $\phi \approx(f a / D)^{2 / 3}$ For $f^{1 / 4} N^{3 / 4} \ll D / a$, the "1n-plane » radius of the star is thus $R / a \approx f^{1 / 3} N(a / D)^{1 / 3}$ and its free energy is $F / k T \approx f^{7 / 3} N(a / D)^{4 / 3}$.

As was discussed, the strong adsorption regime gives nse, in two dimensions, to a Sombrero structure Because of the geometry we always have $f_{\mathrm{ads}}=2$. These must form a contınuous array of $D$ blobs leading to an "1n-plane " radius of $R \approx\left(N / g_{\mathrm{a}}\right) D \approx N d^{1 / 2} a$. For $f \gg 2$, the free arms retain the structure and the radius of a free star The adsorption free energy in this regime is

$$
F_{\mathrm{a}} / k T \approx-\left(N / g_{\mathrm{a}}\right) g_{\mathrm{a}}^{\phi} \delta \approx-N \delta^{2}
$$

The free energy of the free arms is hardly modified when $f \gg 2$. Accordingly, the free energy of the Sombrero structure is

$$
F / k T \approx f^{2}-N \delta^{2}
$$

To characterize the weak adsorption regime we must determine $\theta_{0}$. As before, $\theta_{0}$ is set by two requirements (I) The segment of the truncated star and the absorbed layer are of equal length. (ii) The absorbed layer consists of close packed $D$ blobs incorporating the $n$ monomers in the "missing cap ». The two requirements lead to $\left(n / g_{\mathrm{a}}\right) D \approx R \theta_{0} n$ is given by

$$
n a^{2} \approx \int_{0}^{\theta_{0}} \int_{r(\theta)}^{R_{0}} \phi(r) r \mathrm{~d} r \mathrm{~d} \theta \approx a^{2 / 3} f^{2 / 3}\left(\left(R_{0}^{4 / 3}-H^{4 / 3}\right) \theta_{0} \approx a^{2} f^{2 / 3}\left(R_{0} / a\right)^{4 / 3} \theta_{0}^{3}\right) .
$$

Since $R / a \approx f^{1 / 4} N^{3 / 4}, n$ is

$$
n \approx f N \theta_{0}^{3}
$$

leading to

$$
\theta_{0} \approx f^{-3 / 8} N^{-1 / 8} \delta^{-1 / 4}
$$

The adsorption free energy is thus

$$
F_{\mathrm{a}} / k T \approx-\left(n / g_{\mathrm{a}}\right) g_{\mathrm{a}}^{\phi} \delta \approx-f^{-1 / 8} N^{5 / 8} \delta^{5 / 4}
$$


an the total free energy of the star is

$$
F / k T \approx f^{2}-f^{-1 / 8} N^{5 / 8} \delta^{5 / 4} .
$$

The cross over between the strong and weak adsorption regimes occurs when the number of adsorbed monomers per star is of order $N$, 1.e, when $f \theta_{0}^{3} \approx 1$ or

$$
\delta \approx f^{-1 / 6} N^{-1 / 2} \text {. }
$$

\section{References}

[1] Daoud M., Cotton J P, J Phys. France 43 (1982) 531

[2] Witten T, Pincus P, Macromolecules 19 (1986) 2509

[3] Birsthein T M, Zhulina E B, Polymer 25 (1984) 1453.

[4] HALPERIN A, Macromolecules 20 (1987) 2943

[5] Marques C., Joanny J., Leibler L, Macromolecules 21 (1988) 1051

[6] NAPPER D, «Polymenc Stabilization of Colloidal Dispersions》 (Academic Press, New York) 1983

[7] Elaissari A., Pefferkorn E, $J$ Colloid Interface $S c i$ (in press)

[8] HALPERIN A. AleXANDER S, Macromolecules 20 (1987) 1146

[9] Eisenriegler E, Kremer K, Binder K, J Chem Phys 77 (1982) 6296.

[10] De Gennes P G, Pincus P, J. Phys Lett France 44 (1983) L241.

[11] Bouchaud E., Daoud M, J Phys France 48 (1987) 1991

[12] De Gennes P G, Adv Collotd. Interface Scl 27 (1987) 189

[13] Cohen-Stuart M, Cosgrove T, Vincent B, Adv Colloid Interface Sci 24 (1986) 143

[14] BINDER K, Private Communication (1990)

[15] OONO K, Binder K, J Phys France 49 (1988) 1329.

[16] Eisenriegler E., $J$ Chem Phys 79 (1983) 1052 